\title{
Hypergroups Related to a Pair of Compact Hypergroups
}

\author{
Herbert HEYER $\dagger^{\dagger^{1}}$, Satoshi KAWAKAMI ${ }^{2}$, Tatsuya TSURII $\dagger^{3}$ and Satoe YAMANAKA $\dagger^{4}$ \\ $\dagger^{1}$ Universität Tübingen, Mathematisches Institut, \\ Auf der Morgenstelle 10, 72076, Tübingen, Germany \\ E-mail: herbert.heyer@uni-tuebingen.de \\ $\dagger^{2}$ Nara University of Education, Department of Mathematics, \\ Takabatake-cho Nara, 630-8528, Japan \\ E-mail:kawakami@nara-edu.ac.jp \\ $\dagger^{3}$ Osaka Prefecture University, 1-1 Gakuen-cho, Nakaku, Sakai Osaka, 599-8531, Japan \\ E-mail:dw301003@edu.osakafu-u.ac.jp \\ $\dagger^{4}$ Nara Women's University, Faculty of Science, Kitauoya-higashimachi, Nara, 630-8506, Japan \\ E-mail:s.yamanaka516@gmail.com
}

Received June 02, 2016, in final form November 10, 2016; Published online November 18, 2016

http://dx.doi.org/10.3842/SIGMA.2016.111

\begin{abstract}
The purpose of the present paper is to investigate a hypergroup associated with irreducible characters of a compact hypergroup $H$ and a closed subhypergroup $H_{0}$ of $H$ with $\left|H / H_{0}\right|<+\infty$. The convolution of this hypergroup is introduced by inducing irreducible characters of $H_{0}$ to $H$ and by restricting irreducible characters of $H$ to $H_{0}$. The method of proof relies on the notion of an induced character and an admissible hypergroup pair.
\end{abstract}

Key words: hypergroup; induced character; semi-direct product hypergroup; admissible hypergroup pair

2010 Mathematics Subject Classification: 22D30; 22F50; 20N20; 43A62

\section{Introduction}

The aim of the present paper is to contribute to the largely open problem of establishing a structure theory of hypergroups. Hypergroups are locally compact spaces on which the bounded measures convolve similar to the group case. The origin of the notion of hypergroup or generalized translation structure goes back to J. Delsarte and B.M. Levitan, the special class of double coset hypergroups appears already in the work of G. Frobenius.

There exists an axiomatic approach to hypergroups initiated by Charles F. Dunkl [2, 3], R.I. Jewett [13], and R. Spector [15], which lead to an extensive harmonic analysis of hypergroups. For the historical background of the theory we just refer to R.I. Jewett's fundamental paper [13] and the monograph [1] by W.R. Bloom and H. Heyer. In fact, hypergroups arose in the theory of second order differential equations and developed to be of significant applicability in probability theory where the hypergroup convolution of measure reflects a stochastic operation in the basic space of the hypergroup. Nowadays hypergroup structures are studied within various frameworks from non-commutative duality of groups to quantum groups and bimodules.

Since every investigation of the structures of hypergroups is oriented on the search of new, probably large examples, aspects of a partial solution to the structure problem are extension of hypergroups [4, 6], a cohomology theory for hypergroups [7] and imprimitivity of representations of hypergroups [8]. There are interesting results on hypergroup structures arising from 
dual objects of a hypergroup including the group case [5]. Recent research on the structure of hypergroups relies on the application of induced characters [11, 12], hyperfields [9] and compact hypergroup pairs [10]. At this point we can outline our new results.

Let $H$ be a strong compact hypergroup satisfying the second axiom of countability, and let $H_{0}$ be a subhypergroup of $H$ with $\left|H / H_{0}\right|<+\infty$. By $\mathbb{Z}_{q}(2)$ we denote the $q$-deformation of $\mathbb{Z}(2)$, and the hats on $H$ and $H_{0}$ signify their duals. In [11] the notion of an induced character of a finite-dimensional representation of $H$ was introduced and studied in detail. The results obtained in that paper enable us in the present work to discuss character hypergroups of the type $\mathcal{K}\left(\hat{H} \cup \widehat{H_{0}}, \mathbb{Z}_{q}(2)\right)$ which generalize those introduced in [10]. The admissible group pair of [10] will now be replaced by an admissible hypergroup pair, and the hypergroup structure of $\mathcal{K}\left(\hat{H} \cup \widehat{H_{0}}, \mathbb{Z}_{q}(2)\right)$ will be characterized by the hypergroup pair $\left(H, H_{0}\right)$ (Theorem 3.8). Applications to semi-direct product hypergroups follow (Theorem 4.7), and a list of new hypergroups appears in Section 5.

\section{Preliminaries}

In order to facilitate the reader's access to the problem discussed in this paper we recapitulate the notion of a hypergroup and of a few often applied facts. Details of the theory of hypergroups and standard examples can be found in the seminal paper [13] of R.I. Jewett and in the monograph [1] of W.R. Bloom and H. Heyer.

For a given locally compact (Hausdorff) space $X$ we denote by $C^{b}(X)$ the space of bounded continuous functions on $X$, and by $C_{c}(X)$ and $C_{0}(X)$ its subspaces of functions with compact support or of functions vanishing at infinity respectively. For each compact subset $K$ of $X$ let $C_{K}(X)$ be the subset of functions $f \in C_{c}(X)$ with $\operatorname{supp}(f) \subset K$. By $M(X)$ we denote the set of Radon measures on $X$ defined as linear functionals on $C_{c}(X)$ whose restriction to each $C_{K}(X)$ is continuous with respect to the topology of uniform convergence. $M^{b}(X)$ symbolizes the set of bounded measures on $X$. In fact, $M^{b}(X)$ is the dual of the Banach space $C_{0}(X)$, and it is furnished with the norm

$$
\mu \mapsto\|\mu\|:=\sup \{|\mu(f)|:\|f\| \leq 1\} .
$$

Moreover, we shall refer to the subspaces $M_{c}(X)$ and $M^{1}(X)$ of measures with compact support or probability measures on $X$ respectively.

Finally, $M_{c}^{1}(X):=M^{1}(X) \cap M_{c}(X)$. We denote the Dirac measure in $x \in X$ by $\varepsilon_{x}$.

A hypergroup is a locally compact (Hausdorff) space $H$ together with a weakly continuous associative and bilinear convolution $*$ in the Banach space $M^{b}(H)$ satisfying the following axioms:

(HG1) For all $x, y \in H, \varepsilon_{x} * \varepsilon_{y}$ belongs to $M_{c}^{1}(H)$.

(HG2) There exist a neutral element $e \in H$ such that

$$
\varepsilon_{x} * \varepsilon_{e}=\varepsilon_{e} * \varepsilon_{x}=\varepsilon_{x}
$$

for all $x \in H$, and a continuous involution

$$
x \longmapsto x^{-}
$$

in $H$ satisfying

$$
e \in \operatorname{supp}\left(\varepsilon_{x} * \varepsilon_{y}\right) \text { if and only if } y=x^{-}
$$


as well as

$$
\left(\varepsilon_{x} * \varepsilon_{y}\right)^{-}=\varepsilon_{y^{-}} * \varepsilon_{x^{-}}
$$

whenever $x, y \in H$.

(HG3) The mapping

$$
(x, y) \longmapsto \operatorname{supp}\left(\varepsilon_{x} * \varepsilon_{y}\right)
$$

from $H \times H$ into the space of compact subsets of $H$ equipped with the Michael topology is continuous.

As a consequence of the weak continuity and bilinearity the convolution of arbitrary bounded measures on $H$ is uniquely determined by the convolution of Dirac measures. In other words

$$
\mu * \nu=\int_{H} \int_{H} \varepsilon_{x} * \varepsilon_{y} \mu(d x) \nu(d y),
$$

where $\mu, \nu \in M^{b}(H)$.

A hypergroup $H$ is called commutative if its convolution is commutative. Clearly, locally compact groups are hypergroups. Also double coset spaces $G / / L$ arising from Gelfand pairs $(G, L)$ are (commutative) hypergroups. Given a hypergroup $H$ one can introduce subhypergroups, quotient hypergroups, direct and semi-direct product hypergroups (for the latter notion see $[8,18])$, and hypergroup joins.

Every compact hypergroup $H$ has the normalized Haar measure $\omega_{H} \in M(H)$ which is invariant with respect to the translation

$$
f \mapsto f_{x}, \quad x \in H,
$$

where

$$
f_{x}(y):=\varepsilon_{x} * \varepsilon_{y}(f)=\int_{H} f(z)\left(\varepsilon_{x} * \varepsilon_{y}\right)(d z)
$$

for all $y \in H$.

Let $(H, *)$ and $(L, \circ)$ be two hypergroups with convolutions $*$ and $\circ$ as well with neutral elements $\varepsilon_{H}$ and $\varepsilon_{L}$ respectively. A continuous mapping $\varphi: H \rightarrow L$ is called a hypergroup homomorphism if $\varphi\left(\varepsilon_{H}\right)=\varepsilon_{L}$ and if $\varphi$ is the unique linear weakly continuous extension from $M^{b}(H)$ to $M^{b}(L)$ satisfying the following conditions:

(1) $\varphi\left(\varepsilon_{x}\right)=\varepsilon_{\varphi(x)}$,

(2) $\varphi\left(\varepsilon_{x}^{-}\right)=\varphi\left(\varepsilon_{x}\right)^{-}$,

(3) $\varphi\left(\varepsilon_{x} * \varepsilon_{y}\right)=\varepsilon_{\varphi(x)} \circ \varepsilon_{\varphi(y)}$,

whenever $x, y \in H$.

If, in addition, $\varphi$ is a homeomorphism from $H$ onto $L$, it is called an isomorphism from $H$ onto $L$, and in the case $L=H$ it is called an automorphism of $H$. The set $\operatorname{Aut}(H)$ of all automorphisms of $H$ becomes a topological group furnished with the weak topology of $M^{b}(H)$.

An action of a locally compact group $G$ on a hypergroup $H$ is a continuous homomorphism from $G$ into $\operatorname{Aut}(H)$.

Given an action $\alpha$ of $G$ on $H$ there is the notion of a semi-direct product hypergroup $K=$ $H \rtimes_{\alpha} G$ which in general is a non-commutative hypergroup, efficiently applied all over in our work. 
Let $H$ be a hypergroup, and let $\mathcal{H}$ be a (separable) Hilbert space with inner product $\langle\cdot, \cdot\rangle$. By $\mathcal{B}(\mathcal{H})$ we denote the Banach $*$-algebra of bounded linear operator on $\mathcal{H}$. A $*$-homomorphism $\pi: M^{b}(H) \longrightarrow \mathcal{B}(\mathcal{H})$ is called a representation of $H$ if

$$
\pi\left(\varepsilon_{e}\right)=1
$$

and for all $u, v \in \mathcal{H}$ the mapping

$$
\mu \mapsto\langle\pi(\mu) u, v\rangle
$$

is continuous on $M^{b}(H)$.

In the sequel we shall deal with classes of representations and of irreducible representations of $H$ under unitary equivalence.

Now let $H$ be a compact hypergroup with a countable basis of its topology. $\hat{H}$ will denote the set of all equivalence classes of irreducible representations of $H$. $H$ is said to be of strong type if $\hat{H}$ carries a hypergroup structure. If $H$ is commutative, more structure is available. In this case $\hat{H}$ consists of characters of $H$ which are defined as nonvanishing functions $\chi \in C^{b}(H)$ satisfying the equality

$$
\left(\varepsilon_{x} * \varepsilon_{y}^{-}\right)(\chi)=\chi(x) \bar{\chi}(y)
$$

valid for all $x, y \in H$. Once $\hat{H}$ is a hypergroup, the double dual $\hat{\hat{H}}$ can be formed, and the identification $\hat{\hat{H}} \cong H$ defines Pontryagin hypergroups.

Returning to an arbitrary compact hypergroup $H$ and a closed subhypergroup $H_{0}$ of $H$, for a representation $\pi_{0}$ of $H_{0}$ with representing Hilbert space $\mathcal{H}\left(\pi_{0}\right)$ one introduces the representation

$$
\pi:=\operatorname{ind}_{H_{0}}^{H} \pi_{0}
$$

induced by $\pi_{0}$ from $H_{0}$ to $H$ as follows:

$$
\mathcal{H}(\pi):=\left\{\xi \in L^{2}\left(H, \mathcal{H}\left(\pi_{0}\right)\right):\left(\varepsilon_{h_{0}} * \varepsilon_{x}\right)(\xi)=\pi_{0}\left(h_{0}\right) \xi(x) \text { for all } h_{0} \in H_{0}\right\}
$$

and

$$
(\pi(h) \xi)(x):=\left(\varepsilon_{x} * \varepsilon_{h}\right)(\xi)
$$

for all $\xi \in \mathcal{H}(\pi), x, h \in H$.

For further details on induced representations, see $[8,11]$.

\section{Hypergroups related to admissible pairs}

Let $H$ be a strong compact hypergroup which satisfies the second axiom of countability, and $\hat{H}$ its dual. Then

$$
\mathcal{K}(\hat{H})=\{\operatorname{ch}(\pi): \pi \in \hat{H}\}
$$

is a countable discrete commutative hypergroup, where

$$
\operatorname{ch}(\pi)(h)=\frac{1}{\operatorname{dim} \pi} \operatorname{tr}(\pi(h)) .
$$

for all $\pi \in \hat{H}, h \in H$. 
Now, let $H_{0}$ be a subhypergroup of $H$ which is assumed to be also of strong type and such that $\left|H / H_{0}\right|<+\infty$. For $\tau \in \widehat{H_{0}}$ the induced representation $\operatorname{ind}_{H_{0}}^{H} \tau$ of $\tau$ from $H_{0}$ to $H$ is finite-dimensional and decomposes as

$$
\operatorname{ind}_{H_{0}}^{H} \tau \cong \pi_{1} \oplus \cdots \oplus \pi_{m}
$$

where $\pi_{1}, \ldots, \pi_{m} \in \hat{H}(m \geq 1)$. The induced character of $\operatorname{ch}(\tau)$ is defined as

$$
\operatorname{ind}_{H_{0}}^{H} \operatorname{ch}(\tau):=\frac{d\left(\pi_{1}\right)}{d(\pi)} \operatorname{ch}\left(\pi_{1}\right)+\cdots+\frac{d\left(\pi_{m}\right)}{d(\pi)} \operatorname{ch}\left(\pi_{m}\right)
$$

where $d\left(\pi_{j}\right)$ for $j=1, \ldots, m$ is the hyperdimension of $\pi_{j}$ in the sense of Vrem [17] and

$$
d(\pi):=d\left(\pi_{1}\right)+\cdots+d\left(\pi_{m}\right) .
$$

For $\tau_{i}, \tau_{j} \in \widehat{H_{0}}, \operatorname{ch}\left(\tau_{i}\right) \operatorname{ch}\left(\tau_{j}\right) \in M_{c}^{1}\left(\mathcal{K}\left(\widehat{H_{0}}\right)\right)$ such that

$$
\operatorname{ch}\left(\tau_{i}\right) \operatorname{ch}\left(\tau_{j}\right)=a_{1} \operatorname{ch}\left(\tau_{1}\right)+\cdots+a_{\ell} \operatorname{ch}\left(\tau_{\ell}\right),
$$

with $a_{k}>0(k=1, \ldots, \ell)$ and $a_{1}+\cdots+a_{\ell}=1$. Concerning characters induced from $H_{0}$ to $H$ and the following definition see [11].

Definition 3.1. For $\tau_{i}, \tau_{j} \in \widehat{H_{0}}$,

$$
\operatorname{ind}_{H_{0}}^{H}\left(\operatorname{ch}\left(\tau_{i}\right) \operatorname{ch}\left(\tau_{j}\right)\right):=a_{1} \operatorname{ind}_{H_{0}}^{H} \operatorname{ch}\left(\tau_{1}\right)+\cdots+a_{\ell} \operatorname{ind}_{H_{0}}^{H} \operatorname{ch}\left(\tau_{\ell}\right) .
$$

Our main objective of study will be formulated in the subsequent

Definition 3.2. Let $\mathbb{Z}_{q}(2)$ be a hypergroup of order 2 with parameter $q \in(0,1]$. The twisted convolution $*=*_{q}$ on the space

$$
\mathcal{K}\left(\hat{H} \cup \widehat{H_{0}}, \mathbb{Z}_{q}(2)\right):=\left\{(\operatorname{ch}(\pi), \circ),(\operatorname{ch}(\tau), \bullet): \pi \in \hat{H}, \tau \in \widehat{H_{0}}\right\},
$$

associated with $\mathbb{Z}_{q}(2)$ is given as follows:

(1) $\left(\operatorname{ch}\left(\pi_{i}\right), \circ\right) *\left(\operatorname{ch}\left(\pi_{j}\right), \circ\right):=\left(\operatorname{ch}\left(\pi_{i}\right) \operatorname{ch}\left(\pi_{j}\right), \circ\right)$,

(2) $(\operatorname{ch}(\pi), \circ) *(\operatorname{ch}(\tau), \bullet):=\left(\left(\operatorname{res}_{H_{0}}^{H} \operatorname{ch}(\pi)\right) \operatorname{ch}(\tau), \bullet\right)$,

(3) $(\operatorname{ch}(\tau), \bullet) *(\operatorname{ch}(\pi), \circ):=\left(\operatorname{ch}(\tau)\left(\operatorname{res}_{H_{0}}^{H} \operatorname{ch}(\pi)\right), \bullet\right)$,

(4) $\left(\operatorname{ch}\left(\tau_{i}\right), \bullet\right) *\left(\operatorname{ch}\left(\tau_{j}\right), \bullet\right):=q\left(\operatorname{ind}_{H_{0}}^{H}\left(\operatorname{ch}\left(\tau_{i}\right) \operatorname{ch}\left(\tau_{j}\right)\right), \circ\right)+(1-q)\left(\operatorname{ch}\left(\tau_{i}\right) \operatorname{ch}\left(\tau_{j}\right), \bullet\right)$.

For details on deformation of hypergroups see [14].

Definition 3.3. Let $\left(H, H_{0}\right)$ be a pair of consisting of a compact hypergroup $H$ and a closed subhypergroup $H_{0}$ of $H$. We call $\left(H, H_{0}\right)$ an admissible hypergroup pair if the following conditions are satisfied:

(1) for $\pi \in \hat{H}$ and $\tau \in \widehat{H_{0}}$

$$
\operatorname{ind}_{H_{0}}^{H}\left(\left(\operatorname{res}_{H_{0}}^{H} \operatorname{ch}(\pi)\right) \operatorname{ch}(\tau)\right)=\operatorname{ch}(\pi) \operatorname{ind}_{H_{0}}^{H} \operatorname{ch}(\tau),
$$

(2) for $\tau \in \widehat{H_{0}}$

$$
\operatorname{res}_{H_{0}}^{H}\left(\operatorname{ind}_{H_{0}}^{H} \operatorname{ch}(\tau)\right)=\operatorname{ch}(\tau) \operatorname{res}_{H_{0}}^{H}\left(\operatorname{ind}_{H_{0}}^{H} \operatorname{ch}\left(\tau_{0}\right)\right),
$$

where $\tau_{0}$ is the trivial representation of $H_{0}$. 
Remark 3.4. If a pair $\left(G, G_{0}\right)$ consisting of a compact group and a closed subgroup $G_{0}$ is admissible in the sense of [10], then it is an admissible hypergroup pair.

Lemma 3.5. If $\left(H, H_{0}\right)$ is an admissible hypergroup pair, the following formulae hold:

(1) for $\pi \in \hat{H}$ and $\tau_{i}, \tau_{j} \in \widehat{H_{0}}$

$$
\operatorname{ind}_{H_{0}}^{H}\left(\left(\operatorname{res}_{H_{0}}^{H} \operatorname{ch}(\pi)\right) \operatorname{ch}\left(\tau_{i}\right) \operatorname{ch}\left(\tau_{j}\right)\right)=\operatorname{ch}(\pi) \operatorname{ind}_{H_{0}}^{H}\left(\operatorname{ch}\left(\tau_{i}\right) \operatorname{ch}\left(\tau_{j}\right)\right),
$$

(2) for $\tau_{i}, \tau_{j} \in \widehat{H_{0}}$

$$
\operatorname{res}_{H_{0}}^{H}\left(\operatorname{ind}_{H_{0}}^{H} \operatorname{ch}\left(\tau_{i}\right) \operatorname{ch}\left(\tau_{j}\right)\right)=\operatorname{ch}\left(\tau_{i}\right) \operatorname{ch}\left(\tau_{j}\right) \operatorname{res}_{H_{0}}^{H}\left(\operatorname{ind}_{H_{0}}^{H} \operatorname{ch}\left(\tau_{0}\right)\right)
$$

Proof. It is easy to see the desired formulae by the definition of $\operatorname{ind}_{H_{0}}^{H}\left(\operatorname{ch}\left(\tau_{i}\right) \operatorname{ch}\left(\tau_{j}\right)\right)$.

Proposition 3.6. If a strong compact hypergroup $H$ together with a strong subhypergroup $H_{0}$ of $H$ with $\left|H / H_{0}\right|<+\infty$ forms an admissible hypergroup pair, then the following associativity relations hold. For $\pi_{i}, \pi_{j}, \pi_{k}, \pi \in \hat{H}$ and $\tau_{i}, \tau_{j}, \tau_{k}, \tau \in \widehat{H_{0}}$ :

$$
\begin{aligned}
& \left(\left(\operatorname{ch}\left(\pi_{i}\right), \circ\right) *\left(\operatorname{ch}\left(\pi_{j}\right), \circ\right)\right) *\left(\operatorname{ch}\left(\pi_{k}\right), \circ\right)=\left(\operatorname{ch}\left(\pi_{i}\right), \circ\right) *\left(\left(\operatorname{ch}\left(\pi_{j}\right), \circ\right) *\left(\operatorname{ch}\left(\pi_{k}\right), \circ\right)\right), \\
& \left((\operatorname{ch}(\tau), \bullet) *\left(\operatorname{ch}\left(\pi_{i}\right), \circ\right)\right) *\left(\operatorname{ch}\left(\pi_{j}\right), \circ\right)=(\operatorname{ch}(\tau), \bullet) *\left(\left(\operatorname{ch}\left(\pi_{i}\right), \circ\right) *\left(\operatorname{ch}\left(\pi_{j}\right), \circ\right)\right), \\
& \left(\left(\operatorname{ch}\left(\tau_{i}\right), \bullet\right) *\left(\operatorname{ch}\left(\tau_{j}\right), \bullet\right)\right) *(\operatorname{ch}(\pi), \circ)=\left(\operatorname{ch}\left(\tau_{i}\right), \bullet\right) *\left(\left(\operatorname{ch}\left(\tau_{j}\right), \bullet\right) *(\operatorname{ch}(\pi), \circ)\right), \\
& \left(\left(\operatorname{ch}\left(\tau_{i}\right), \bullet\right) *\left(\operatorname{ch}\left(\tau_{j}\right), \bullet\right)\right) *\left(\operatorname{ch}\left(\tau_{k}\right), \bullet\right)=\left(\operatorname{ch}\left(\tau_{i}\right), \bullet\right) *\left(\left(\operatorname{ch}\left(\tau_{j}\right), \bullet\right) *\left(\operatorname{ch}\left(\tau_{k}\right), \bullet\right)\right) .
\end{aligned}
$$

Proof. $(A 1)$ is clear by the assumption that $H$ is strong, i.e., $\mathcal{K}(\hat{H})$ is a hypergroup.

(A2) For $\tau \in \widehat{H_{0}}$ and $\pi_{i}, \pi_{j} \in \hat{H}$,

$$
\begin{aligned}
\left((\operatorname{ch}(\tau), \bullet) *\left(\operatorname{ch}\left(\pi_{i}\right), \circ\right)\right) *\left(\operatorname{ch}\left(\pi_{j}\right), \circ\right) & =\left(\operatorname{ch}(\tau) \operatorname{res}_{H_{0}}^{H} \operatorname{ch}\left(\pi_{i}\right), \bullet\right) *\left(\operatorname{ch}\left(\pi_{j}\right), \circ\right) \\
& =\left(\operatorname{ch}(\tau)\left(\operatorname{res}_{H_{0}}^{H} \operatorname{ch}\left(\pi_{i}\right)\right)\left(\operatorname{res}_{H_{0}}^{H} \operatorname{ch}\left(\pi_{j}\right)\right), \bullet\right) .
\end{aligned}
$$

On the other hand,

$$
\begin{aligned}
(\operatorname{ch}(\tau), \bullet) *\left(\left(\operatorname{ch}\left(\pi_{i}\right), \circ\right) *\left(\operatorname{ch}\left(\pi_{j}\right), \circ\right)\right) & =(\operatorname{ch}(\tau), \bullet) *\left(\operatorname{ch}\left(\pi_{i}\right) \operatorname{ch}\left(\pi_{j}\right), \circ\right) \\
& =\left(\operatorname{ch}(\tau) \operatorname{res}_{H_{0}}^{H}\left(\operatorname{ch}\left(\pi_{i}\right) \operatorname{ch}\left(\pi_{j}\right)\right), \bullet\right) \\
& =\left(\operatorname{ch}(\tau)\left(\operatorname{res}_{H_{0}}^{H} \operatorname{ch}\left(\pi_{i}\right)\right)\left(\operatorname{res}_{H_{0}}^{H} \operatorname{ch}\left(\pi_{j}\right)\right), \bullet\right) .
\end{aligned}
$$

(A3) For $\tau_{i}, \tau_{j} \in \widehat{H_{0}}$ and $\pi \in \hat{H}$,

$$
\begin{aligned}
& \left(\left(\operatorname{ch}\left(\tau_{i}\right), \bullet\right) *\left(\operatorname{ch}\left(\tau_{j}\right), \bullet\right)\right) *(\operatorname{ch}(\pi), \circ) \\
& \quad=\left(q\left(\operatorname{ind}_{H_{0}}^{H}\left(\operatorname{ch}\left(\tau_{i}\right) \operatorname{ch}\left(\tau_{j}\right)\right), \circ\right)+(1-q)\left(\operatorname{ch}\left(\tau_{i}\right) \operatorname{ch}\left(\tau_{j}\right), \bullet\right)\right) *(\operatorname{ch}(\pi), \circ) \\
& \quad=q\left(\operatorname{ind}_{H_{0}}^{H}\left(\operatorname{ch}\left(\tau_{i}\right) \operatorname{ch}\left(\tau_{j}\right)\right), \circ\right) *(\operatorname{ch}(\pi), \circ)+(1-q)\left(\operatorname{ch}\left(\tau_{i}\right) \operatorname{ch}\left(\tau_{j}\right), \bullet\right) *(\operatorname{ch}(\pi), \circ) \\
& \quad=q\left(\operatorname{ind}_{H_{0}}^{H}\left(\operatorname{ch}\left(\tau_{i}\right) \operatorname{ch}\left(\tau_{j}\right)\right) \operatorname{ch}(\pi), \circ\right)+(1-q)\left(\operatorname{ch}\left(\tau_{i}\right) \operatorname{ch}\left(\tau_{j}\right) \operatorname{res}_{H_{0}}^{H} \operatorname{ch}(\pi), \bullet\right) \\
& \quad=q\left(\operatorname{ind}_{H_{0}}^{H}\left(\operatorname{ch}\left(\tau_{i}\right) \operatorname{ch}\left(\tau_{j}\right) \operatorname{res}_{H_{0}}^{H} \operatorname{ch}(\pi)\right), \circ\right)+(1-q)\left(\operatorname{ch}\left(\tau_{i}\right) \operatorname{ch}\left(\tau_{j}\right) \operatorname{res}_{H_{0}}^{H} \operatorname{ch}(\pi), \bullet\right) \\
& \quad=\left(\operatorname{ch}\left(\tau_{i}\right), \bullet\right) *\left(\operatorname{ch}\left(\tau_{j}\right) \operatorname{res}_{H_{0}}^{H} \operatorname{ch}(\pi), \bullet\right) \\
& \quad=\left(\operatorname{ch}\left(\tau_{i}\right), \bullet\right) *\left(\left(\operatorname{ch}\left(\tau_{j}\right), \bullet\right) *(\operatorname{ch}(\pi), \circ)\right) .
\end{aligned}
$$

(A4) For $\tau_{i}, \tau_{j}, \tau_{k} \in \widehat{H_{0}}$

$$
\left(\left(\operatorname{ch}\left(\tau_{i}\right), \bullet\right) *\left(\operatorname{ch}\left(\tau_{j}\right), \bullet\right)\right) *\left(\operatorname{ch}\left(\tau_{k}\right), \bullet\right)
$$




$$
\begin{aligned}
= & \left(q\left(\operatorname{ind}_{H_{0}}^{H}\left(\operatorname{ch}\left(\tau_{i}\right) \operatorname{ch}\left(\tau_{j}\right)\right), \circ\right)+(1-q)\left(\operatorname{ch}\left(\tau_{i}\right) \operatorname{ch}\left(\tau_{j}\right), \bullet\right)\right) *\left(\operatorname{ch}\left(\tau_{k}\right), \bullet\right) \\
= & q\left(\operatorname{ind}_{H_{0}}^{H}\left(\operatorname{ch}\left(\tau_{i}\right) \operatorname{ch}\left(\tau_{j}\right)\right), \circ\right) *\left(\operatorname{ch}\left(\tau_{k}\right), \bullet\right)+(1-q)\left(\operatorname{ch}\left(\tau_{i}\right) \operatorname{ch}\left(\tau_{j}\right), \bullet\right) *\left(\operatorname{ch}\left(\tau_{k}\right), \bullet\right) \\
= & q\left(\operatorname{res}_{H_{0}}^{H}\left(\operatorname{ind}_{H_{0}}^{H}\left(\operatorname{ch}\left(\tau_{i}\right) \operatorname{ch}\left(\tau_{j}\right)\right)\right) \operatorname{ch}\left(\tau_{k}\right), \bullet\right) \\
& +(1-q) q\left(\operatorname{ind}_{H_{0}}^{H}\left(\operatorname{ch}\left(\tau_{i}\right) \operatorname{ch}\left(\tau_{j}\right) \operatorname{ch}\left(\tau_{k}\right)\right), \circ\right)+(1-q)^{2}\left(\operatorname{ch}\left(\tau_{i}\right) \operatorname{ch}\left(\tau_{j}\right) \operatorname{ch}\left(\tau_{k}\right), \bullet\right) \\
= & q\left(\operatorname{res}_{H_{0}}^{H}\left(\operatorname{ind}_{H_{0}}^{H} \operatorname{ch}\left(\tau_{0}\right)\right)\left(\operatorname{ch}\left(\tau_{i}\right) \operatorname{ch}\left(\tau_{j}\right) \operatorname{ch}\left(\tau_{k}\right)\right), \bullet\right) \\
& +(1-q) q\left(\operatorname{ind}_{H_{0}}^{H}\left(\operatorname{ch}\left(\tau_{i}\right) \operatorname{ch}\left(\tau_{j}\right) \operatorname{ch}\left(\tau_{k}\right)\right), \circ\right)+(1-q)^{2}\left(\operatorname{ch}\left(\tau_{i}\right) \operatorname{ch}\left(\tau_{j}\right) \operatorname{ch}\left(\tau_{k}\right), \bullet\right) .
\end{aligned}
$$

This implies the associativity:

$$
\left(\left(\operatorname{ch}\left(\tau_{i}\right), \bullet\right) *\left(\operatorname{ch}\left(\tau_{j}\right), \bullet\right)\right) *\left(\operatorname{ch}\left(\tau_{k}\right), \bullet\right)=\left(\operatorname{ch}\left(\tau_{i}\right), \bullet\right) *\left(\left(\operatorname{ch}\left(\tau_{j}\right), \bullet\right) *\left(\operatorname{ch}\left(\tau_{k}\right), \bullet\right)\right) .
$$

Proposition 3.7. Let $H$ be a strong compact hypergroup and $H_{0}$ a subhypergroup of $H$ which is also of strong type and such that $\left|H / H_{0}\right|<+\infty$. If $\mathcal{K}\left(\hat{H} \cup \widehat{H_{0}}, \mathbb{Z}_{q}(2)\right)$ is a hypergroup, then $\left(H, H_{0}\right)$ is an admissible hypergroup pair.

Proof. (1) By the associativity (A3) we have

$$
(\operatorname{ch}(\tau), \bullet) *\left(\left(\operatorname{ch}\left(\tau_{0}\right), \bullet\right) *(\operatorname{ch}(\pi), \circ)\right)=\left((\operatorname{ch}(\tau), \bullet) *\left(\operatorname{ch}\left(\tau_{0}\right), \bullet\right)\right) *(\operatorname{ch}(\pi), \circ),
$$

and the formulae

$$
\begin{aligned}
& (\operatorname{ch}(\tau), \bullet) *\left(\left(\operatorname{ch}\left(\tau_{0}\right), \bullet\right) *(\operatorname{ch}(\pi), \circ)\right) \\
& \quad=q\left(\operatorname{ind}_{H_{0}}^{H}\left(\operatorname{ch}(\tau) \operatorname{res}_{H_{0}}^{H} \operatorname{ch}(\pi)\right), \circ\right)+(1-q)\left(\operatorname{ch}(\tau) \operatorname{res}_{H_{0}}^{H} \operatorname{ch}(\pi), \bullet\right)
\end{aligned}
$$

and

$$
\begin{aligned}
& \left((\operatorname{ch}(\tau), \bullet) *\left(\operatorname{ch}\left(\tau_{0}\right), \bullet\right)\right) *(\operatorname{ch}(\pi), \circ) \\
& \quad=q\left(\left(\operatorname{ind}_{H_{0}}^{H} \operatorname{ch}(\tau)\right) \operatorname{ch}(\pi), \circ\right)+(1-q)\left(\operatorname{ch}(\tau) \operatorname{res}_{H_{0}}^{H} \operatorname{ch}(\pi), \bullet\right) .
\end{aligned}
$$

Hence we obtain the admissibility condition (1)

$$
\operatorname{ind}_{H_{0}}^{H}\left(\left(\operatorname{res}_{H_{0}}^{H} \operatorname{ch}(\pi)\right) \operatorname{ch}(\tau)\right)=\operatorname{ch}(\pi) \operatorname{ind}_{H_{0}}^{H} \operatorname{ch}(\tau) .
$$

(2) By the associativity $(A 4)$

$$
\begin{aligned}
& (\operatorname{ch}(\tau), \bullet) *\left(\left(\operatorname{ch}\left(\tau_{0}\right), \bullet\right) *\left(\operatorname{ch}\left(\tau_{0}\right), \bullet\right)\right) \\
& \quad=\left((\operatorname{ch}(\tau), \bullet) *\left(\operatorname{ch}\left(\tau_{0}\right), \bullet\right)\right) *\left(\operatorname{ch}\left(\tau_{0}\right), \bullet\right),\left((\operatorname{ch}(\tau), \bullet) *\left(\operatorname{ch}\left(\tau_{0}\right), \bullet\right)\right) *\left(\operatorname{ch}\left(\tau_{0}\right), \bullet\right) \\
& \quad=q\left(\operatorname{res}_{H_{0}}^{H}\left(\operatorname{ind}_{H_{0}}^{H} \operatorname{ch}(\tau), \bullet\right)+(1-q) q\left(\operatorname{ind}_{H_{0}}^{H} \operatorname{ch}(\tau)\right), \circ\right)+(1-q)^{2}(\operatorname{ch}(\tau), \bullet),
\end{aligned}
$$

and

$$
\begin{aligned}
& (\operatorname{ch}(\tau), \bullet) *\left(\left(\operatorname{ch}\left(\tau_{0}\right), \bullet\right) *\left(\operatorname{ch}\left(\tau_{0}\right), \bullet\right)\right) \\
& \quad=q\left(\operatorname{ch}(\tau) \operatorname{res}_{H_{0}}^{H}\left(\operatorname{ind}_{H_{0}}^{H} \operatorname{ch}\left(\tau_{0}\right)\right), \bullet\right)+(1-q) q\left(\operatorname{ind}_{H_{0}}^{H} \operatorname{ch}(\tau), \circ\right)+(1-q)^{2}(\operatorname{ch}(\tau), \bullet) .
\end{aligned}
$$

Hence we obtain the admissibility condition (2)

$$
\operatorname{res}_{H_{0}}^{H}\left(\operatorname{ind}_{H_{0}}^{H} \operatorname{ch}(\tau)\right)=\operatorname{ch}(\tau) \operatorname{res}_{H_{0}}^{H}\left(\operatorname{ind}_{H_{0}}^{H} \operatorname{ch}\left(\tau_{0}\right)\right) .
$$

Theorem 3.8. Let $H$ be a strong compact hypergroup and $H_{0}$ a subhypergroup of $H$ which is also of strong type and such that $\left|H / H_{0}\right|<+\infty$. Then $\mathcal{K}\left(\hat{H} \cup \widehat{H_{0}}, \mathbb{Z}_{q}(2)\right)$ is a hypergroup if and only if $\left(H, H_{0}\right)$ is an admissible hypergroup pair. 
Proof. If $\left(H, H_{0}\right)$ is an admissible hypergroup pair, then the associativity relations $(A 1),(A 2)$, $(A 3)$ and $(A 4)$ are a consequence of Proposition 3.6. It is easy to check the remaining axioms of a hypergroup for $\mathcal{K}\left(\hat{H} \cup \widehat{H_{0}}, \mathbb{Z}_{q}(2)\right)$. The converse statement follows from Proposition 3.7.

\section{Remark 3.9.}

(1) The above $\mathcal{K}\left(\hat{H} \cup \widehat{H_{0}}, \mathbb{Z}_{q}(2)\right)$ is a discrete commutative (at most countable) hypergroup such that the sequence

$$
1 \longrightarrow \mathcal{K}(\hat{H}) \longrightarrow \mathcal{K}\left(\hat{H} \cup \widehat{H}_{0}, \mathbb{Z}_{q}(2)\right) \longrightarrow \mathbb{Z}_{q}(2) \longrightarrow 1
$$

is exact.

(2) If $H_{0}=H$, then $\mathcal{K}\left(\hat{H} \cup \widehat{H_{0}}, \mathbb{Z}_{q}(2)\right)$ is the direct product hypergroup $\mathcal{K}(\hat{H}) \times \mathbb{Z}_{q}(2)$.

(3) If $H$ is a finite hypergroup and $H_{0}=\left\{h_{0}\right\}$ where $h_{0}$ is unit of $H$, then $\mathcal{K}\left(\hat{H} \cup \widehat{H_{0}}, \mathbb{Z}_{q}(2)\right)$ is the hypergroup join $\mathcal{K}(\hat{H}) \vee \mathbb{Z}_{q}(2)$.

(4) If $H$ is a compact commutative hypergroup of strong type and $H_{0}$ is a closed subhypergroup of $H$ with $\left|H / H_{0}\right|<+\infty$. Then $\left(H, H_{0}\right)$ is always an admissible hypergroup pair and $\mathcal{K}\left(\hat{H} \cup \widehat{H_{0}}, \mathbb{Z}_{q}(2)\right)$ is a hypergroup. For more details see [9].

\section{Semi-direct product hypergroups}

We consider a non-commutative compact hypergroup, namely the semi-direct product hypergroup $K:=H \rtimes_{\alpha} G$, where $\alpha$ is an action of a compact group $G$ on a compact commutative hypergroup $H$ of strong type. For a representation $\pi$ of $K$ we denote the restrictions of $\pi$ to $H$ and $G$ by $\rho$ and $\tau$ respectively. We shall write $\pi=\rho \odot \tau$ expressing

$$
\pi(h, g)=\rho(h) \tau(g)
$$

for all $h \in H, g \in G$. The action $\hat{\alpha}$ of $G$ on $\hat{H}$ induced by $\alpha$ is given by

$$
\hat{\alpha}_{g}(\chi)(h):=\chi\left(\alpha_{g}^{-1}(h)\right),
$$

whenever $\chi \in \hat{H}, g \in G, h \in H$. Let

$$
G(\chi):=\left\{g \in G: \hat{\alpha}_{g}(\chi)=\chi\right\}
$$

be the stabilizer of $\chi \in \hat{H}$ under the action $\hat{\alpha}$ of $G$ on $\hat{H}$.

Proposition 4.1. Any irreducible representation $\pi$ of $K=H \rtimes_{\alpha} G$ is given by

$$
\pi=\pi^{(\chi, \tau)}:=\operatorname{ind}_{H \rtimes_{\alpha} G(\chi)}^{H \rtimes_{\alpha} G}(\chi \odot \tau),
$$

where $\chi \in \hat{H}, \tau \in \widehat{G(\chi)}$.

Proof. This statement is obtained by an application of the Mackey machine as stated in Theorem 7.1 of [8].

We denote the orbit of $\chi \in \hat{H}$ by $O(\chi)$ under the action $\hat{\alpha}$ of $G$ on $\hat{H}$, i.e.,

$$
O(\chi):=\left\{\hat{\alpha}_{g}(\chi): g \in G\right\}
$$


Then the representation $\rho^{\chi}$ associated with $O(\chi)$ is defined by

$$
\rho^{\chi}:=\int_{O(\chi)}^{\oplus} \sigma \mu(d \sigma)
$$

where $\mu$ is the $\hat{\alpha}$-invariant probability measure supporting the orbit $O(\chi)$ of $\chi$ in $\hat{H}$. We denote the space $\left\{\rho^{\chi}: \chi \in \hat{H}\right\}$ by $\hat{H}^{\hat{\alpha}}$. By the assumption that $H$ is strong we see that $\hat{H}^{\hat{\alpha}}$ is a hypergroup which is called the orbital hypergroup of $\hat{H}$ under the action $\hat{\alpha}$. Here we note that the irreducible representation $\pi^{(\chi, \tau)}$ of $K=H \rtimes_{\alpha} G$ is written as $\pi^{(\chi, \tau)}=\rho^{\chi} \odot u^{\tau}$, where the representation $u^{\tau}$ of $G$ is given by

$$
u^{\tau}=\operatorname{ind}_{G(\chi)}^{G} \tau
$$

Moreover we note that the hyperdimension $d(\pi)$ of $\pi=\pi^{(\chi, \tau)}$ is

$$
d(\pi):=w(\chi) \operatorname{dim} \pi=w\left(\rho^{\chi}\right) \operatorname{dim} \tau,
$$

where $w(\chi)$ and $w\left(\rho^{\chi}\right)=w(\chi)|O(\chi)|$ denote the weights of $\chi \in \hat{H}$ and $\rho^{\chi} \in \hat{H}^{\hat{\alpha}}$ respectively.

Proposition 4.2. For a representation $\pi_{0}=\chi \odot \tau$ of $K_{0}=H \rtimes_{\alpha} G(\chi)$, where $\chi \in \hat{H}, \tau \in \widehat{G(\chi)}$, the character of the induced representation $\pi^{(\chi, \tau)}=\operatorname{ind}_{K_{0}}^{K} \pi_{0}$ of $\pi_{0}$ takes the form

$$
\operatorname{ch}\left(\operatorname{ind}_{K_{0}}^{K} \pi_{0}\right)(h, g)=\int_{G} \chi\left(\alpha_{s}(h)\right) \operatorname{ch}(\tau)\left(s g s^{-1}\right) 1_{G(\chi)}\left(s g s^{-1}\right) \omega_{G}(d s) .
$$

Moreover,

$$
\left(\operatorname{ind}_{K_{0}}^{K} \operatorname{ch}\left(\pi_{0}\right)\right)(h, g)=\int_{G} \chi\left(\alpha_{s}(h)\right) \operatorname{ch}(\tau)\left(s g s^{-1}\right) 1_{G(\chi)}\left(s g s^{-1}\right) \omega_{G}(d s),
$$

hence

$$
\operatorname{ind}_{K_{0}}^{K} \operatorname{ch}\left(\pi_{0}\right)=\operatorname{ch}\left(\operatorname{ind}_{K_{0}}^{K} \pi_{0}\right)=\operatorname{ch}\left(\pi^{(\chi, \tau)}\right) .
$$

Proof. By an application of the character formulae as proved in Proposition 4.3 and Theorem 4.5 of [11], we obtain the desired formulae.

Proposition 4.3 ([11, Theorem 4.6]). Let $H$ be a finite commutative hypergroup of strong type. Then the induced representation $\pi=\operatorname{ind}_{G}^{H \rtimes_{\alpha} G} \tau$ of an irreducible representation $\tau$ of $G$ to $H \rtimes_{\alpha} G$ is finite-dimensional, and it is decomposed as

$$
\pi \cong \sum_{\rho^{\chi \in \hat{H}^{\hat{\alpha}}}} \pi^{(\chi, \tau)} .
$$

The character $\operatorname{ch}(\pi)$ of $\pi$ is

$$
\operatorname{ch}(\pi)=\sum_{\rho^{\chi \in \hat{H}^{\hat{\alpha}}}} \frac{w\left(\rho^{\chi}\right)}{w(\hat{H})} \operatorname{ch}\left(\pi^{(\chi, \tau)}\right) .
$$

Definition $4.4([5])$. The action $\alpha$ of $G$ on $H$ is said to satisfy the regularity condition (or is called regular) provided

$$
G\left(\chi_{i}\right) \cap G\left(\chi_{j}\right) \subset G\left(\chi_{k}\right)
$$

for all $\chi_{k} \in \hat{H}$ such that $\chi_{k} \in \operatorname{supp}\left(\delta_{\chi_{i}} \hat{*} \delta_{\chi_{j}}\right)$ whenever $\chi_{i}, \chi_{j} \in \hat{H}, k, i, j \in\{0,1, \ldots, n\}$ and $\hat{*}$ symbolizes the convolution on $\hat{H}$. 
Lemma 4.5 ([5, Lemma 3.1]). If the action $\alpha$ satisfies the regularity condition, then the character set $\mathcal{K}\left(\widehat{H \rtimes_{\alpha} G}\right)$ of the semi-direct product hypergroup $H \rtimes_{\alpha} G$ is a commutative hypergroup.

For $g \in G$, put

$$
\hat{H}(g):=\{\sigma \in \hat{H}: g \in G(\sigma)\}=\left\{\sigma \in \hat{H}: \hat{\alpha}_{g}(\sigma)=\sigma\right\} .
$$

Proposition 4.6. Let $H$ be a finite commutative hypergroup of strong type and $G$ a compact group. Assume that the action $\alpha$ of $G$ on $H$ satisfies the regularity condition. Then the followings hold:

(1) $\hat{H}(g)$ is a subhypergroup of $\hat{H}$,

(2) for $g, t \in G$ the condition $t g t^{-1} \in G(\sigma)$ implies that $\hat{\alpha}_{t}^{-1}(\sigma) \in \hat{H}(g)$,

(3) for $\tau \in \hat{G}$

$$
\left(\operatorname{ind}_{G}^{H \rtimes_{\alpha} G} \operatorname{ch}(\tau)\right)(h, g)=\frac{w(\hat{H}(g))}{w(\hat{H})} \omega_{\hat{H}(g)}(h) \cdot \operatorname{ch}(\tau)(g),
$$

where $\omega_{\hat{H}(g)}$ is the normalized Haar measure of $\hat{H}(g)$,

(4) for $\tau \in \hat{G}$

$$
\operatorname{res}_{G}^{H \rtimes_{\alpha} G}\left(\operatorname{ind}_{G}^{H \rtimes_{\alpha} G} \operatorname{ch}(\tau)\right)(g)=\frac{w(\hat{H}(g))}{w(\hat{H})} \cdot \operatorname{ch}(\tau)(g) .
$$

Proof. (1) It is clear that $\sigma^{-} \in \hat{H}(g)$ for $\sigma \in \hat{H}(g)$. We show that $\operatorname{supp}\left(\sigma_{i} \hat{*} \sigma_{j}\right) \subset \hat{H}(g)$ for $\sigma_{i}, \sigma_{j} \in \hat{H}(g)$. The condition $\sigma_{i}, \sigma_{j} \in \hat{H}(g)$ implies that $g \in G\left(\sigma_{i}\right) \cap G\left(\sigma_{j}\right)$. Take $\sigma \in$ $\operatorname{supp}\left(\sigma_{i} \hat{*} \sigma_{j}\right)$. Then by the regularity condition

$$
G\left(\sigma_{i}\right) \cap G\left(\sigma_{j}\right) \subset G(\sigma)
$$

we see that $g \in G(\sigma)$ which implies that $\sigma \in \hat{H}(g)$.

(2) By the condition $t g t^{-1} \in G(\sigma)$ we see that $\hat{\alpha}_{t g t^{-1}}(\sigma)=\sigma$. Then we obtain

$$
\hat{\alpha}_{t}\left(\hat{\alpha}_{g}\left(\hat{\alpha}_{t^{-1}}(\sigma)\right)\right)=\sigma,
$$

so that

$$
\hat{\alpha}_{g}\left(\hat{\alpha}_{t}^{-1}(\sigma)\right)=\hat{\alpha}_{t}^{-1}(\sigma),
$$

which means that $\hat{\alpha}_{t}^{-1}(\sigma) \in \hat{H}(g)$.

(3) Applying Propositions 4.2 and 4.3 we obtain for $\tau \in \hat{G}$

$$
\begin{aligned}
\left(\operatorname{ind}_{G}^{H \rtimes_{\alpha} G} \operatorname{ch}(\tau)\right)(h, g) & =\sum_{\rho^{\chi \in \hat{H}^{\hat{\alpha}}}} \frac{w\left(\rho^{\chi}\right)}{w(\hat{H})} \operatorname{ch}\left(\pi^{(\chi, \tau)}\right)(h, g) \\
& =\sum_{\rho^{\chi \in \hat{H}^{\hat{\alpha}}}} \frac{w\left(\rho^{\chi}\right)}{w(\hat{H})} \int_{G} \chi\left(\alpha_{s}(h)\right) \operatorname{ch}(\tau)\left(s g s^{-1}\right) 1_{G(\chi)}\left(\operatorname{sgs}^{-1}\right) d \omega_{G}(s) \\
& =\sum_{\rho^{\chi \in \hat{H}^{\hat{\alpha}}}} \frac{w\left(\rho^{\chi}\right)}{w(\hat{H})} \int_{G} \chi\left(\alpha_{s}(h)\right) 1_{G(\chi)}\left(\operatorname{sgs}^{-1}\right) d \omega_{G}(s) \cdot \operatorname{ch}(\tau)(g) \\
& =\sum_{\rho^{\chi \in \hat{H}^{\hat{\alpha}}}} \frac{|O(\chi)| w(\chi)}{w(\hat{H})} \cdot \frac{1}{|O(\chi)|} \sum_{\sigma \in O(\chi)} \sigma(h) 1_{G(\sigma)}(g) \cdot \operatorname{ch}(\tau)(g)
\end{aligned}
$$




$$
\begin{aligned}
& =\sum_{\sigma \in \hat{H}} \frac{w(\sigma)}{w(\hat{H})} \sigma(h) 1_{G(\sigma)}(g) \cdot \operatorname{ch}(\tau)(g) \\
& =\frac{w(\hat{H}(g))}{w(\hat{H})} \sum_{\sigma \in \hat{H}(g)} \frac{w(\sigma)}{w(\hat{H}(g))} \sigma(h) \cdot \operatorname{ch}(\tau)(g) \\
& =\frac{w(\hat{H}(g))}{w(\hat{H})} \omega_{\hat{H}(g)}(h) \cdot \operatorname{ch}(\tau)(g) .
\end{aligned}
$$

(4) For $\tau \in \hat{G}$

$$
\begin{aligned}
\operatorname{res}_{G}^{H \rtimes_{\alpha} G}\left(\operatorname{ind}_{G}^{H \rtimes_{\alpha} G} \operatorname{ch}(\tau)\right)(g) & =\left(\operatorname{ind}_{G}^{H \rtimes_{\alpha} G} \operatorname{ch}(\tau)\right)\left(h_{0}, g\right) \\
& =\frac{w(\hat{H}(g))}{w(\hat{H})} \omega_{\hat{H}(g)}\left(h_{0}\right) \cdot \operatorname{ch}(\tau)(g)=\frac{w(\hat{H}(g))}{w(\hat{H})} \cdot \operatorname{ch}(\tau)(g) .
\end{aligned}
$$

Theorem 4.7. Let $H$ be a finite commutative hypergroup of strong type and $G$ a compact group. Suppose that the action $\alpha$ of $G$ on $H$ satisfies the regularity condition. Then the pair $\left(H \rtimes_{\alpha} G, G\right)$ is an admissible hypergroup pair and $\mathcal{K}\left(\widehat{H \rtimes_{\alpha}} G \cup \hat{G}, \mathbb{Z}_{q}(2)\right)$ is a discrete commutative hypergroup.

Proof. By the Mackey machine an irreducible representation $\pi$ of $K=H \rtimes_{\alpha} G$ is given by

$$
\pi=\pi^{\left(\chi, \tau_{1}\right)}=\operatorname{ind}_{H \rtimes_{\alpha} G(\chi)}^{H \rtimes_{\alpha} G}\left(\chi \odot \tau_{1}\right),
$$

where $\chi \in \hat{H}, \tau_{1} \in \widehat{G(\chi)}$ and

$$
\pi^{\left(\chi, \tau_{1}\right)}(h, g)=\int_{G} \chi\left(\alpha_{s}(h)\right) \operatorname{ch}\left(\tau_{1}\right)\left(\operatorname{sgs}^{-1}\right) 1_{G(\chi)}\left(\operatorname{sgs}^{-1}\right) \omega_{G}(d s),
$$

by Propositions 4.1 and 4.2 .

(1) For $\tau \in \hat{G}$ and $\pi^{\left(\chi, \tau_{1}\right)} \in \hat{K}=\widehat{H \rtimes_{\alpha} G}$, applying Proposition 4.6,

$$
\begin{aligned}
\left(\operatorname{ind}_{G}^{H} \rtimes_{\alpha} G\right. & \left.\operatorname{ch}(\tau) \cdot \operatorname{ch}\left(\pi^{\left(\chi, \tau_{1}\right)}\right)\right)(h, g)=\left(\operatorname{ind}_{G}^{H \rtimes_{\alpha} G} \operatorname{ch}(\tau)\right)(h, g) \operatorname{ch}\left(\pi^{\left(\chi, \tau_{1}\right)}\right)(h, g) \\
= & \frac{w(\hat{H}(g))}{w(\hat{H})} \omega_{\hat{H}(g)}(h) \cdot \operatorname{ch}(\tau)(g) \int_{G} \chi\left(\alpha_{s}(h)\right) \operatorname{ch}\left(\tau_{1}\right)\left(s g s^{-1}\right) 1_{G(\chi)}\left(s g s^{-1}\right) \omega_{G}(d s) \\
& =\frac{w(\hat{H}(g))}{w(\hat{H})} \cdot \operatorname{ch}(\tau)(g) \int_{G} \omega_{\hat{H}(g)}(h) \hat{\alpha}_{s}^{-1}(\chi)(h) \operatorname{ch}\left(\tau_{1}\right)\left(s g s^{-1}\right) 1_{G(\chi)}\left(s g s^{-1}\right) \omega_{G}(d s) \\
& =\frac{w(\hat{H}(g))}{w(\hat{H})} \cdot \operatorname{ch}(\tau)(g) \int_{G}\left(\omega_{\hat{H}(g)} \hat{\alpha}_{s}^{-1}(\chi)\right)(h) \operatorname{ch}\left(\tau_{1}\right)\left(s g s^{-1}\right) 1_{G(\chi)}\left(s g s^{-1}\right) \omega_{G}(d s) \\
& =\frac{w(\hat{H}(g))}{w(\hat{H})} \cdot \operatorname{ch}(\tau)(g) \int_{G} \omega_{\hat{H}(g)}(h) \operatorname{ch}\left(\tau_{1}\right)\left(s g s^{-1}\right) 1_{G(\chi)}\left(s g s^{-1}\right) \omega_{G}(d s) \\
& =\frac{w(\hat{H}(g))}{w(\hat{H})} \omega_{\hat{H}(g)}(h) \cdot \operatorname{ch}(\tau)(g) \int_{G} \operatorname{ch}\left(\tau_{1}\right)\left(s g s^{-1}\right) 1_{G(\chi)}\left(s g s^{-1}\right) \omega_{G}(d s) \\
& =\left(\operatorname{ind}_{G}^{H \rtimes_{\alpha} G}\left(\operatorname{ch}(\tau)\left(\operatorname{res}_{G}^{H \rtimes_{\alpha} G} \operatorname{ch}(\pi)\right)\right)\right)(h, g) .
\end{aligned}
$$

Hence we obtain the admissibility condition (1).

(2) For $\tau \in \hat{G}$, applying Proposition 4.6,

$$
\begin{aligned}
\operatorname{res}_{G}^{H \rtimes_{\alpha} G}\left(\operatorname{ind}_{G}^{H \rtimes_{\alpha} G} \operatorname{ch}(\tau)\right)(g) & =\frac{w(\hat{H}(g))}{w(\hat{H})} \cdot \operatorname{ch}(\tau)(g)=\frac{w(\hat{H}(g))}{w(\hat{H})} \cdot \operatorname{ch}\left(\tau_{0}\right)(g) \cdot \operatorname{ch}(\tau)(g) \\
& =\operatorname{res}_{G}^{H \rtimes_{\alpha} G}\left(\operatorname{ind}_{G}^{H \rtimes_{\alpha} G} \tau_{0}\right)(g) \cdot \operatorname{ch}(\tau)(g) .
\end{aligned}
$$

Hence we see the admissibility condition (2).

By Theorem 3.8 we see that $\mathcal{K}\left(\widehat{H \rtimes_{\alpha}} G \cup \hat{G}, \mathbb{Z}_{q}(2)\right)$ is a discrete commutative hypergroup. 


\section{Examples of $\mathcal{K}\left(\hat{H} \cup \widehat{H}_{0}, \mathbb{Z}_{q}(2)\right)$}

In this section we illustrate some special case of

$$
\mathcal{K}\left(\hat{H} \cup \widehat{H_{0}}, \mathbb{Z}_{q}(2)\right)=\left\{\left(\operatorname{ch}\left(\pi_{i}\right), \circ\right),\left(\operatorname{ch}\left(\tau_{j}\right), \bullet\right): \pi_{i} \in \hat{H}, \tau_{j} \in \widehat{H_{0}}\right\} .
$$

We denote $\left(\operatorname{ch}\left(\pi_{i}\right), \circ\right)$ for $\pi_{i} \in \hat{H}$ by $\gamma_{i}$ and $\left(\operatorname{ch}\left(\tau_{j}\right), \bullet\right)$ for $\tau_{j} \in \widehat{H_{0}}$ by $\rho_{j}$ hereafter.

Example 5.1. Let $H=\mathbb{Z}_{p}(2)=\left\{h_{0}, h_{1}\right\}$ and $H_{0}=\left\{h_{0}\right\}$ where $h_{0}$ is unit of $H$. In this case $\hat{H}=\left\{\pi_{0}, \pi_{1}\right\}$ and $\widehat{H_{0}}=\left\{\tau_{0}\right\}$. The Frobenius diagram (we refer to [10]) is

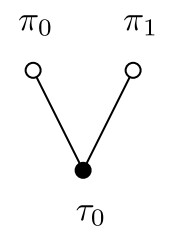

The structure equations of $\mathcal{K}\left(\widehat{\mathbb{Z}_{p}(2)} \cup \widehat{\left\{h_{0}\right\}}, \mathbb{Z}_{q}(2)\right)=\left\{\gamma_{0}, \gamma_{1}, \rho_{0}\right\}$ are

$$
\gamma_{1} \gamma_{1}=p \gamma_{0}+(1-p) \gamma_{1}, \quad \rho_{0} \rho_{0}=\frac{q}{2} \gamma_{0}+\frac{q}{2} \gamma_{1}+(1-q) \rho_{0}, \quad \gamma_{1} \rho_{0}=\rho_{0} .
$$

We note that $\mathcal{K}\left(\widehat{\mathbb{Z}_{p}(2)} \cup \widehat{\left\{h_{0}\right\}}, \mathbb{Z}_{q}(2)\right) \cong \mathbb{Z}_{p}(2) \vee \mathbb{Z}_{q}(2)$ and $(p, q)$-deformations of the hypergroup associated with Dynkin diagram $A_{3}$ refer to Sunder-Wildberger [16].

Example 5.2. Let $H=\mathbb{Z}_{p}(3)=\left\{h_{0}, h_{1}, h_{2}\right\}$ and $H=\left\{h_{0}\right\}$ In this case $\hat{H}=\left\{\pi_{0}, \pi_{1}, \pi_{2}\right\}$ and $\widehat{H_{0}}=\left\{\tau_{0}\right\}$. The Frobenius diagram is

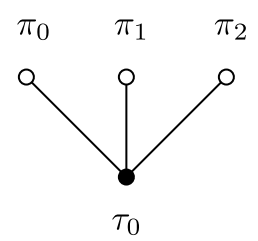

The structure equations of $\mathcal{K}\left(\widehat{\mathbb{Z}_{p}(3)} \cup \widehat{\left\{h_{0}\right\}}, \mathbb{Z}_{q}(2)\right)=\left\{\gamma_{0}, \gamma_{1}, \gamma_{2}, \rho_{0}\right\}$ are

$$
\begin{aligned}
& \gamma_{1} \gamma_{1}=\frac{1-p}{2} \gamma_{1}+\frac{1+p}{2} \gamma_{2}, \quad \gamma_{2} \gamma_{2}=\frac{1+p}{2} \gamma_{1}+\frac{1-p}{2} \gamma_{2}, \\
& \gamma_{1} \gamma_{2}=p \gamma_{0}+\frac{1-p}{2} \gamma_{1}+\frac{1-p}{2} \gamma_{2}, \quad \rho_{0} \rho_{0}=\frac{q}{3} \gamma_{0}+\frac{q}{3} \gamma_{1}+\frac{q}{3} \gamma_{2}+(1-q) \rho_{0}, \\
& \gamma_{1} \rho_{0}=\gamma_{2} \rho_{0}=\rho_{0} .
\end{aligned}
$$

We note that $\mathcal{K}\left(\widehat{\mathbb{Z}_{p}(3)} \cup \widehat{\left\{h_{0}\right\}}, \mathbb{Z}_{q}(2)\right) \cong \mathbb{Z}_{p}(3) \vee \mathbb{Z}_{q}(2)$ and these hypergroups are $(p, q)$ deformations of the hypergroup associated with Dynkin diagram $D_{4}$ constructed by SunderWildberger [16].

Example 5.3. Let $H=\mathbb{Z}_{(p, r)}(4)=\left\{h_{0}, h_{1}, h_{2}, h_{3}\right\}$ and $H_{0}=\left\{h_{0}\right\}$. In this case $\hat{H}=\left\{\pi_{0}, \pi_{1}\right.$, $\left.\pi_{2}, \pi_{3}\right\}$ and $\widehat{H_{0}}=\left\{\tau_{0}\right\}$. The Frobenius diagram is

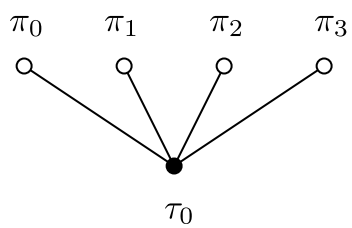


The structure equations of $\mathcal{K}\left(\widehat{\mathbb{Z}_{(p, r)}(4)} \cup \widehat{\left\{h_{0}\right\}}, \mathbb{Z}_{q}(2)\right)=\left\{\gamma_{0}, \gamma_{1}, \gamma_{2}, \gamma_{3}, \rho_{0}\right\}$ are

$$
\begin{array}{ll}
\gamma_{1} \gamma_{1}=\gamma_{3} \gamma_{3}=\frac{1-p}{2} \gamma_{1}+p \gamma_{2}+\frac{1-p}{2} \gamma_{3}, \quad \gamma_{2} \gamma_{2}=r \gamma_{0}+(1-r) \gamma_{2}, \\
\gamma_{1} \gamma_{2}=\frac{1-r}{2} \gamma_{1}+\frac{1+r}{2} \gamma_{3}, & \gamma_{1} \gamma_{3}=\frac{2 p r}{1+r} \gamma_{0}+\frac{1-p}{2} \gamma_{1}+\frac{p-p r}{1+r} \gamma_{2}+\frac{1-p}{2} \gamma_{3}, \\
\gamma_{2} \gamma_{3}=\frac{1+r}{2} \gamma_{1}+\frac{1-r}{2} \gamma_{3}, & \rho_{0} \rho_{0}=\frac{q}{4} \gamma_{0}+\frac{q}{4} \gamma_{1}+\frac{q}{4} \gamma_{2}+\frac{q}{4} \gamma_{3}+(1-q) \rho_{0}, \\
\gamma_{1} \rho_{0}=\gamma_{2} \rho_{0}=\gamma_{3} \rho_{0}=\rho_{0} . &
\end{array}
$$

We note that $\mathcal{K}\left(\widehat{\mathbb{Z}_{(p, r)}(4)} \cup \widehat{\left\{h_{0}\right\}}, \mathbb{Z}_{q}(2)\right) \cong \mathbb{Z}_{(p, r)}(4) \vee \mathbb{Z}_{q}(2)$.

Example 5.4. Let $H=\mathbb{Z}_{(p, r)}(4)=\left\{h_{0}, h_{1}, h_{2}, h_{3}\right\}$ and $H_{0}=\mathbb{Z}_{p}(2)=\left\{h_{0}, h_{2}\right\}$. In this case $\hat{H}=\left\{\pi_{0}, \pi_{1}, \pi_{2}, \pi_{3}\right\}$ and $\widehat{H_{0}}=\left\{\tau_{0}, \tau_{1}\right\}$. The Frobenius diagram is

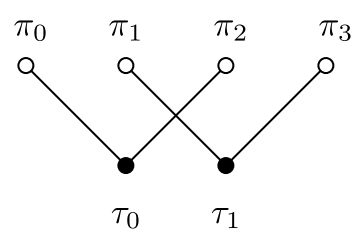

The structure equations of $\mathcal{K}\left(\widehat{\mathbb{Z}_{(p, r)}(4)} \cup \widehat{\mathbb{Z}_{p}(2)}, \mathbb{Z}_{q}(2)\right)=\left\{\gamma_{0}, \gamma_{1}, \gamma_{2}, \gamma_{3}, \rho_{0}, \rho_{1}\right\}$ are

$$
\begin{aligned}
& \gamma_{1} \gamma_{1}=\gamma_{3} \gamma_{3}=\frac{1-p}{2} \gamma_{1}+p \gamma_{2}+\frac{1-p}{2} \gamma_{3}, \quad \gamma_{2} \gamma_{2}=r \gamma_{0}+(1-r) \gamma_{2}, \\
& \gamma_{1} \gamma_{2}=\frac{1-r}{2} \gamma_{1}+\frac{1+r}{2} \gamma_{3}, \quad \gamma_{1} \gamma_{3}=\frac{2 p r}{1+r} \gamma_{0}+\frac{1-p}{2} \gamma_{1}+\frac{p-p r}{1+r} \gamma_{2}+\frac{1-p}{2} \gamma_{3}, \\
& \gamma_{2} \gamma_{3}=\frac{1+r}{2} \gamma_{1}+\frac{1-r}{2} \gamma_{3}, \quad \rho_{0} \rho_{0}=\rho_{1} \rho_{1}=\frac{q}{2} \gamma_{0}+\frac{q}{2} \gamma_{2}+(1-q) \rho_{0}, \\
& \rho_{0} \rho_{1}=\frac{q}{2} \gamma_{1}+\frac{q}{2} \gamma_{3}+(1-q) \rho_{1}, \quad \gamma_{1} \rho_{0}=\gamma_{3} \rho_{0}=\gamma_{2} \rho_{1}=\rho_{1}, \\
& \gamma_{1} \rho_{1}=\gamma_{3} \rho_{1}=\gamma_{2} \rho_{0}=\rho_{0} .
\end{aligned}
$$

Example 5.5. Let $H=S_{p}(3)=\mathbb{Z}_{p}(3) \rtimes_{\alpha} \mathbb{Z}_{2}$ and $H_{0}=\mathbb{Z}_{2}$. In this case $\hat{H}=\left\{\pi_{0}, \pi_{1}, \pi_{2}\right\}$ $\left(\operatorname{dim} \pi_{2}=2\right)$ and $\widehat{H_{0}}=\left\{\tau_{0}, \tau_{1}\right\}$. The Frobenius diagram is

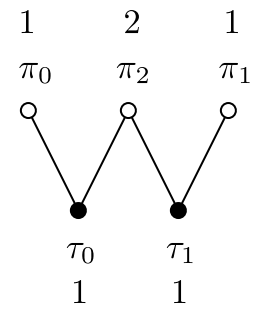

The structure equations of $\mathcal{K}\left(\widehat{S_{p}(3)} \cup \widehat{\mathbb{Z}_{2}}, \mathbb{Z}_{q}(2)\right)=\left\{\gamma_{0}, \gamma_{1}, \gamma_{2}, \rho_{0}, \rho_{1}\right\}$ are

$$
\begin{aligned}
& \gamma_{1} \gamma_{1}=\gamma_{0}, \quad \frac{p}{4} \gamma_{0}+\frac{p}{4} \gamma_{1}+\left(1-\frac{p}{2}\right) \gamma_{2}, \quad \gamma_{1} \gamma_{2}=\gamma_{2}, \\
& \rho_{0} \rho_{0}=\rho_{1} \rho_{1}=\frac{q}{3} \gamma_{0}+\frac{2 q}{3} \gamma_{2}+(1-q) \rho_{0}, \quad \rho_{0} \rho_{1}=\frac{q}{3} \gamma_{1}+\frac{2 q}{3} \gamma_{2}+(1-q) \rho_{1}, \\
& \gamma_{1} \rho_{0}=\rho_{1}, \quad \gamma_{1} \rho_{1}=\rho_{0}, \quad \gamma_{2} \rho_{0}=\gamma_{2} \rho_{1}=\frac{1}{2} \rho_{0}+\frac{1}{2} \rho_{1} .
\end{aligned}
$$

We note that these hypergroups are $(p, q)$-deformations of the hypergroup associated with Dynkin diagram $A_{5}$ refer to Sunder-Wildberger [16]. 
Example 5.6. Let $H=D_{(p, r)}(4)=\mathbb{Z}_{(p, r)}(4) \rtimes_{\alpha} \mathbb{Z}_{2}$ and $H_{0}=\mathbb{Z}_{2}$. In this case $\hat{H}=\left\{\pi_{0}, \pi_{1}, \pi_{2}, \pi_{3}\right.$, $\left.\pi_{4}\right\}\left(\operatorname{dim} \pi_{4}=2\right)$ and $\widehat{H_{0}}=\left\{\tau_{0}, \tau_{1}\right\}$. The Frobenius diagram is

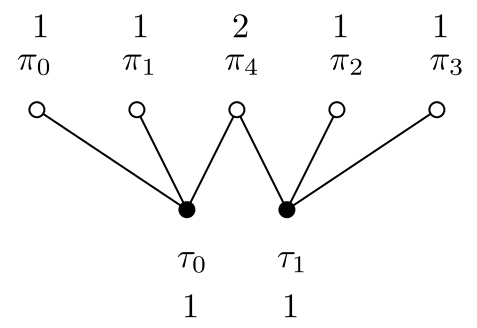

The structure equations of $\mathcal{K}\left(\widehat{D_{(p, r)}(4)} \cup \widehat{\mathbb{Z}_{2}}, \mathbb{Z}_{q}(2)\right)=\left\{\gamma_{0}, \gamma_{1}, \gamma_{2}, \gamma_{3}, \gamma_{4}, \rho_{0}, \rho_{1}\right\}$ are

$$
\begin{aligned}
& \gamma_{1} \gamma_{1}=\gamma_{0}, \quad \gamma_{2} \gamma_{2}=\gamma_{3} \gamma_{3}=r \gamma_{0}+(1-r) \gamma_{2}, \\
& \gamma_{4} \gamma_{4}=\frac{p r}{2(1+r)} \gamma_{0}+\frac{p r}{2(1+r)} \gamma_{1}+\frac{p}{2(1+r)} \gamma_{2}+\frac{p}{2(1+r)} \gamma_{3}+(1-p) \gamma_{4}, \\
& \gamma_{1} \gamma_{2}=\gamma_{3}, \quad \gamma_{1} \gamma_{3}=\gamma_{2}, \quad \gamma_{1} \gamma_{4}=\gamma_{2} \gamma_{4}=\gamma_{3} \gamma_{4}=\gamma_{4}, \quad \gamma_{2} \gamma_{3}=r \gamma_{1}+(1-r) \gamma_{3}, \\
& \rho_{0} \rho_{0}=\rho_{1} \rho_{1}=\frac{q}{4} \gamma_{0}+\frac{q}{4} \gamma_{1}+\frac{q}{2} \gamma_{4}+(1-q) \rho_{0}, \quad \rho_{0} \rho_{1}=\frac{q}{4} \gamma_{2}+\frac{q}{4} \gamma_{3}+\frac{q}{2} \gamma_{4}+(1-q) \rho_{1}, \\
& \gamma_{1} \rho_{0}=\gamma_{2} \rho_{1}=\gamma_{3} \rho_{1}=\rho_{0}, \quad \gamma_{1} \rho_{1}=\gamma_{2} \rho_{0}=\gamma_{3} \rho_{0}=\rho_{1}, \quad \gamma_{4} \rho_{0}=\gamma_{4} \rho_{1}=\frac{1}{2} \rho_{0}+\frac{1}{2} \rho_{1} .
\end{aligned}
$$

Example 5.7. Let $H=A_{4}=\left(\mathbb{Z}_{2} \times \mathbb{Z}_{2}\right) \rtimes_{\alpha} \mathbb{Z}_{3}$ and $H_{0}=\mathbb{Z}_{3}$. In this case $\hat{H}=\left\{\pi_{0}, \pi_{1}, \pi_{2}, \pi_{3}\right\}$ $\left(\operatorname{dim} \pi_{3}=3\right)$ and $\widehat{H_{0}}=\left\{\tau_{0}, \tau_{1}, \tau_{2}\right\}$. The Frobenius diagram is

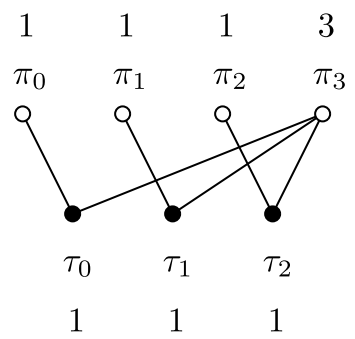

The structure equations of $\mathcal{K}\left(\widehat{A_{4}} \cup \widehat{\mathbb{Z}_{3}}, \mathbb{Z}_{q}(2)\right)=\left\{\gamma_{0}, \gamma_{1}, \gamma_{2}, \gamma_{3}, \gamma_{4}, \rho_{0}, \rho_{1}, \rho_{2}\right\}$ are

$$
\begin{array}{ll}
\gamma_{1} \gamma_{1}=\gamma_{2}, & \gamma_{2} \gamma_{2}=\gamma_{1}, \quad \gamma_{3} \gamma_{3}=\frac{1}{9} \gamma_{0}+\frac{1}{9} \gamma_{1}+\frac{1}{9} \gamma_{2}+\frac{2}{3} \gamma_{3}, \quad \gamma_{1} \gamma_{2}=\gamma_{0}, \\
\gamma_{1} \gamma_{3}=\gamma_{3}, & \gamma_{2} \gamma_{3}=\gamma_{3}, \quad \rho_{0} \rho_{0}=\rho_{1} \rho_{2}=\frac{q}{4} \gamma_{0}+\frac{3 q}{4} \gamma_{3}+(1-q) \rho_{0}, \\
\rho_{1} \rho_{1}=\rho_{0} \rho_{2}=\frac{q}{4} \gamma_{2}+\frac{3 q}{4} \gamma_{3}+(1-q) \rho_{2}, \quad \rho_{2} \rho_{2}=\rho_{0} \rho_{1}=\frac{q}{4} \gamma_{1}+\frac{3 q}{4} \gamma_{3}+(1-q) \rho_{1}, \\
\gamma_{1} \rho_{0}=\rho_{1}, \quad \gamma_{2} \rho_{0}=\gamma_{1} \rho_{1}=\rho_{2}, \quad \gamma_{2} \rho_{1}=\rho_{0}, \\
\gamma_{3} \rho_{0}=\gamma_{3} \rho_{1}=\gamma_{3} \rho_{2}=\frac{1}{3} \rho_{0}+\frac{1}{3} \rho_{1}+\frac{1}{3} \rho_{2} .
\end{array}
$$

Example 5.8. Let $H=S_{4}=A_{4} \rtimes_{\alpha} \mathbb{Z}_{2}$ and $H_{0}=\mathbb{Z}_{2}$. In this case $\hat{H}=\left\{\pi_{0}, \pi_{1}, \pi_{2}, \pi_{3}, \pi_{4}\right\}$ and $\widehat{H_{0}}=\left\{\tau_{0}, \tau_{1}\right\}$. The Frobenius diagram is

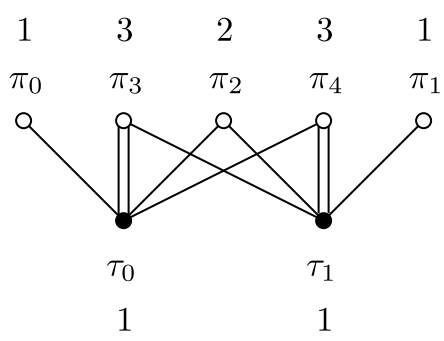


The structure equations of $\mathcal{K}\left(\widehat{S_{4}} \cup \widehat{\mathbb{Z}_{2}}, \mathbb{Z}_{q}(2)\right)=\left\{\gamma_{0}, \gamma_{1}, \gamma_{2}, \gamma_{3}, \gamma_{4}, \rho_{0}, \rho_{1}\right\}$ are

$$
\begin{aligned}
& \gamma_{1} \gamma_{1}=\gamma_{0}, \quad \gamma_{2} \gamma_{2}=\frac{1}{4} \gamma_{0}+\frac{1}{4} \gamma_{1}+\frac{1}{2} \gamma_{2}, \\
& \gamma_{3} \gamma_{3}=\gamma_{4} \gamma_{4}=\frac{1}{9} \gamma_{0}+\frac{2}{9} \gamma_{2}+\frac{1}{3} \gamma_{3}+\frac{1}{3} \gamma_{4}, \\
& \gamma_{1} \gamma_{2}=\gamma_{2}, \quad \gamma_{1} \gamma_{3}=\gamma_{4}, \quad \gamma_{1} \gamma_{4}=\gamma_{3}, \quad \gamma_{2} \gamma_{3}=\gamma_{2} \gamma_{4}=\frac{1}{2} \gamma_{3}+\frac{1}{2} \gamma_{4}, \\
& \gamma_{3} \gamma_{4}=\frac{1}{9} \gamma_{1}+\frac{2}{9} \gamma_{2}+\frac{1}{3} \gamma_{3}+\frac{1}{3} \gamma_{4}, \\
& \rho_{0} \rho_{0}=\rho_{1} \rho_{1}=\frac{q}{12} \gamma_{0}+\frac{q}{6} \gamma_{2}+\frac{q}{2} \gamma_{3}+\frac{q}{4} \gamma_{4}+(1-q) \rho_{0}, \\
& \rho_{0} \rho_{1}=\rho_{1} \rho_{0}=\frac{q}{12} \gamma_{1}+\frac{q}{6} \gamma_{2}+\frac{q}{4} \gamma_{3}+\frac{q}{2} \gamma_{4}+(1-q) \rho_{1}, \\
& \gamma_{0} \rho_{0}=\gamma_{1} \rho_{1}=\rho_{0}, \quad \gamma_{0} \rho_{1}=\gamma_{1} \rho_{0}=\rho_{1}, \quad \gamma_{0}=\gamma_{2} \rho_{1}=\frac{1}{2} \rho_{0}+\frac{1}{2} \rho_{1}, \\
& \gamma_{3} \rho_{0}=\gamma_{4} \rho_{1}=\frac{2}{3} \rho_{0}+\frac{1}{3} \rho_{1}, \quad \gamma_{4} \rho_{0}=\gamma_{3} \rho_{1}=\frac{1}{3} \rho_{0}+\frac{2}{3} \rho_{1} .
\end{aligned}
$$

Example 5.9. Let $H=S_{4}$ and $H_{0}=S_{3}$. In this case $\hat{H}=\left\{\pi_{0}, \pi_{1}, \pi_{2}, \pi_{3}, \pi_{4}\right\}$ and $\widehat{H_{0}}=$ $\left\{\tau_{0}, \tau_{1}, \tau_{2}\right\}$. The Frobenius diagram is

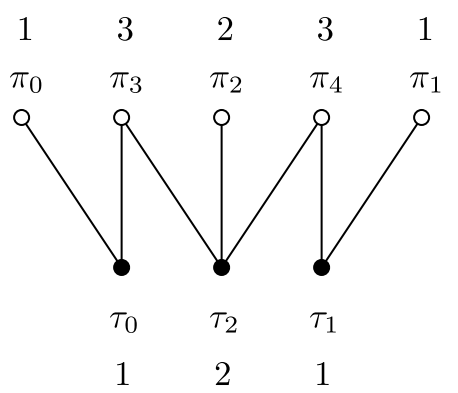

The structure equations of $\mathcal{K}\left(\widehat{S_{4}} \cup \widehat{S_{3}}, \mathbb{Z}_{q}(2)\right)=\left\{\gamma_{0}, \gamma_{1}, \gamma_{2}, \gamma_{3}, \gamma_{4}, \rho_{0}, \rho_{1}, \rho_{2}\right\}$ are

$$
\begin{aligned}
& \gamma_{1} \gamma_{1}=\gamma_{0}, \quad \frac{1}{4} \gamma_{0}+\frac{1}{4} \gamma_{1}+\frac{1}{2} \gamma_{2}, \quad \gamma_{3} \gamma_{3}=\gamma_{4} \gamma_{4}=\frac{1}{9} \gamma_{0}+\frac{2}{9} \gamma_{2}+\frac{1}{3} \gamma_{3}+\frac{1}{3} \gamma_{4}, \\
& \gamma_{1} \gamma_{2}=\gamma_{2}, \quad \gamma_{1} \gamma_{3}=\gamma_{4}, \quad \gamma_{1} \gamma_{4}=\gamma_{3}, \quad \gamma_{2} \gamma_{3}=\gamma_{2} \gamma_{4}=\frac{1}{2} \gamma_{3}+\frac{1}{2} \gamma_{4}, \\
& \gamma_{3} \gamma_{4}=\frac{1}{9} \gamma_{1}+\frac{2}{9} \gamma_{2}+\frac{1}{3} \gamma_{3}+\frac{1}{3} \gamma_{4}, \quad \rho_{0} \rho_{0}=\rho_{1} \rho_{1}=\frac{q}{4} \gamma_{0}+\frac{3 q}{4} \gamma_{3}+(1-q) \rho_{0}, \\
& \rho_{2} \rho_{2}=\frac{q}{16} \gamma_{0}+\frac{q}{16} \gamma_{1}+\frac{q}{8} \gamma_{2}+\frac{3 q}{8} \gamma_{3}+\frac{3 q}{8} \gamma_{4}+\frac{1-q}{4} \rho_{0}+\frac{1-q}{4} \rho_{1}+\frac{1-q}{2} \rho_{2}, \\
& \rho_{1} \rho_{2}=\frac{q}{4} \gamma_{2}+\frac{3 q}{8} \gamma_{3}+\frac{3 q}{8} \gamma_{4}+(1-q) \rho_{2}, \quad \gamma_{0} \rho_{0}=\gamma_{1} \rho_{1}=\rho_{0}, \quad \gamma_{0} \rho_{1}=\gamma_{1} \rho_{0}=\rho_{1}, \\
& \gamma_{0} \rho_{2}=\gamma_{1} \rho_{2}=\gamma_{2} \rho_{0}=\gamma_{2} \rho_{1}=\rho_{2}, \quad \gamma_{3} \rho_{0}=\gamma_{4} \rho_{1}=\frac{1}{3} \rho_{0}+\frac{2}{3} \rho_{2}, \\
& \gamma_{4} \rho_{0}=\gamma_{3} \rho_{1}=\frac{1}{3} \rho_{1}+\frac{2}{3} \rho_{2}, \quad \gamma_{2} \rho_{2}=\frac{1}{4} \rho_{0}+\frac{1}{4} \rho_{1}+\frac{1}{2} \rho_{2}, \\
& \gamma_{3} \rho_{2}=\gamma_{4} \rho_{0}=\frac{1}{6} \rho_{0}+\frac{1}{6} \rho_{1}+\frac{2}{3} \rho_{2} .
\end{aligned}
$$

Example 5.10. Let $H=\mathbb{Z}_{p}(3) \rtimes_{\alpha}\left(\mathbb{Z}_{2} \times \mathbb{T}\right) \cong S_{p}(3) \times \mathbb{T}$ and $H_{0}=\mathbb{Z}_{2} \times \mathbb{T}$. In this case

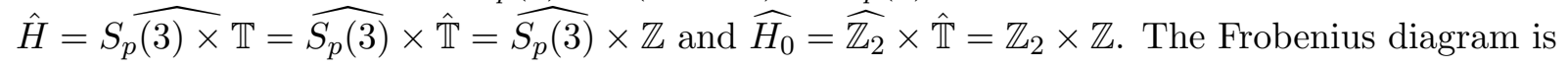




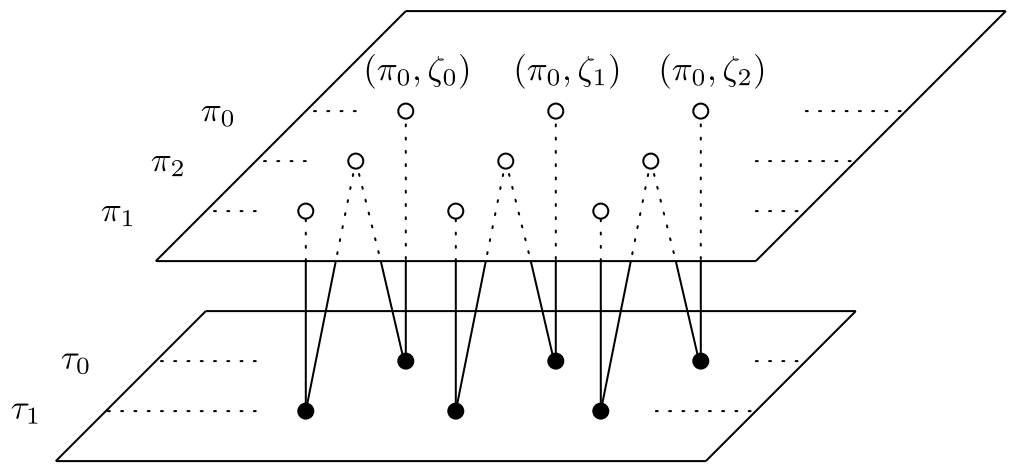

We denote $\left(\operatorname{ch}\left(\left(\pi_{i}, \zeta_{j}\right)\right), \circ\right)$ for $\left(\pi_{i}, \zeta_{j}\right) \in \hat{H}$ by $\left(\gamma_{i}, \xi_{j}\right)$ and $\left(\operatorname{ch}\left(\left(\tau_{k}, \zeta_{\ell}\right)\right), \bullet\right)$ for $\left(\tau_{k}, \zeta_{\ell}\right) \in \widehat{H_{0}}$ by $\left(\rho_{k}, \xi_{\ell}\right)$. The structure equations of

$$
\mathcal{K}\left(\hat{H} \cup \widehat{H_{0}}, \mathbb{Z}_{q}(2)\right)=\left\{\left(\gamma_{i}, \xi_{j}\right),\left(\rho_{k}, \xi_{\ell}\right): i=0,1,2, k=0,1, j, \ell \in \mathbb{Z}\right\}
$$

are

$$
\begin{aligned}
\left(\gamma_{1}, \xi_{j}\right)\left(\gamma_{1}, \xi_{\ell}\right) & =\left(\gamma_{0}, \xi_{j+\ell}\right), \quad\left(\gamma_{1}, \xi_{j}\right)\left(\gamma_{2}, \xi_{\ell}\right)=\left(\gamma_{2}, \xi_{j+\ell}\right), \\
\left(\gamma_{2}, \xi_{j}\right)\left(\gamma_{2}, \xi_{\ell}\right) & =\frac{p}{4}\left(\gamma_{0}, \xi_{j+\ell}\right)+\frac{p}{4}\left(\gamma_{1}, \xi_{j+\ell}\right)+\left(1-\frac{p}{2}\right)\left(\gamma_{2}, \xi_{j+\ell}\right), \\
\left(\rho_{0}, \xi_{j}\right)\left(\rho_{0}, \xi_{\ell}\right) & =\left(\rho_{1}, \xi_{j}\right)\left(\rho_{1}, \xi_{\ell}\right)=\frac{q}{3}\left(\gamma_{0}, \xi_{j+\ell}\right)+\frac{2 q}{3}\left(\gamma_{2}, \xi_{j+\ell}\right)+(1-q)\left(\rho_{0}, \xi_{j+\ell}\right), \\
\left(\rho_{0}, \xi_{j}\right)\left(\rho_{1}, \xi_{\ell}\right) & =\left(\rho_{1}, \xi_{\ell}\right)\left(\rho_{0}, \xi_{j}\right)=\frac{q}{3}\left(\gamma_{1}, \xi_{j+\ell}\right)+\frac{2 q}{3}\left(\gamma_{2}, \xi_{j+\ell}\right)+(1-q)\left(\rho_{1}, \xi_{j+\ell}\right), \\
\left(\gamma_{0}, \xi_{j}\right)\left(\rho_{0}, \xi_{\ell}\right) & =\left(\gamma_{1}, \xi_{j}\right)\left(\rho_{1}, \xi_{\ell}\right)=\left(\rho_{0}, \xi_{j+\ell}\right), \\
\left(\gamma_{0}, \xi_{j}\right)\left(\rho_{1}, \xi_{\ell}\right) & =\left(\gamma_{1}, \xi_{j}\right)\left(\rho_{0}, \xi_{\ell}\right)=\left(\rho_{1}, \xi_{j+\ell}\right), \\
\left(\gamma_{2}, \xi_{j}\right)\left(\rho_{0}, \xi_{\ell}\right) & =\left(\gamma_{2}, \xi_{j}\right)\left(\rho_{1}, \xi_{\ell}\right)=\frac{1}{2}\left(\rho_{0}, \xi_{j+\ell}\right)+\frac{1}{2}\left(\rho_{1}, \xi_{j+\ell}\right) .
\end{aligned}
$$

\section{References}

[1] Bloom W.R., Heyer H., Harmonic analysis of probability measures on hypergroups, de Gruyter Studies in Mathematics, Vol. 20, Walter de Gruyter \& Co., Berlin, 1995.

[2] Dunkl C.F., The measure algebra of a locally compact hypergroup, Trans. Amer. Math. Soc. 179 (1973), $331-348$.

[3] Dunkl C.F., Structure hypergroups for measure algebras, Pacific J. Math. 47 (1973), 413-425.

[4] Heyer H., Katayama Y., Kawakami S., Kawasaki K., Extensions of finite commutative hypergroups, Sci. Math. Jpn. 65 (2007), 373-385.

[5] Heyer H., Kawakami S., Hypergroup structures arising from certain dual objects of a hypergroup, J. Math. Soc. Japan, to appear.

[6] Heyer H., Kawakami S., Extensions of Pontryagin hypergroups, Probab. Math. Statist. 26 (2006), 245-260.

[7] Heyer H., Kawakami S., A cohomology approach to the extension problem for commutative hypergroups, Semigroup Forum 83 (2011), 371-394.

[8] Heyer H., Kawakami S., An imprimitivity theorem for representations of a semi-direct product hypergroup, J. Lie Theory 24 (2014), 159-178.

[9] Heyer H., Kawakami S., Tsurii T., Yamanaka S., A commutative hypergroup associated with a hyperfield, arXiv:1604.04361.

[10] Heyer H., Kawakami S., Tsurii T., Yamanaka S., Hypergroups arising from characters of a compact group and its subgroup, arXiv:1605.03744. 
[11] Heyer H., Kawakami S., Yamanaka S., Characters of induced representations of a compact hypergroup, Monatsh. Math. 179 (2016), 421-440.

[12] Hirai T., Classical method of constructing a complete set of irreducible representations of semidirect product of a compact group with a finite group, Probab. Math. Statist. 33 (2013), 353-362.

[13] Jewett R.I., Spaces with an abstract convolution of measures, Adv. Math. 18 (1975), 1-101.

[14] Kawakami S., Tsurii T., Yamanaka S., Deformations of finite hypergroups, Sci. Math. Japan e-2015 (2015), 2015-21, 11 pages.

[15] Spector R., Aperçu de la théorie des hypergroupes, in Analyse Harmonique sur les groupes de Lie (Sém. Nancy-Strasbourg, 1973-1975), Lecture Notes in Math., Vol. 497, Springer, Berlin, 1975, 643-673.

[16] Sunder V.S., Wildberger N.J., Fusion rule algebras and walks on graphs, in The Proceedings of the Fifth Ramanujan Symposium on Harmonic Analysis, Editor K.R. Parthasarathy, Ramanujan Institute, 1999, 53-80.

[17] Vrem R.C., Harmonic analysis on compact hypergroups, Pacific J. Math. 85 (1979), 239-251.

[18] Willson B., Configurations and invariant nets for amenable hypergroups and related algebras, Trans. Amer. Math. Soc. 366 (2014), 5087-5112. 